\title{
The utility of biomarkers in traumatic brain injury clinical management
}

\author{
Ana Rodríguez-Rodríguez ${ }^{1}$ and Juan José Egea-Guerrero ${ }^{2^{*}}$ \\ See related research by Thelin et al. http://ccforum.biomedcentral.com/articles/10.1186/s13054-016-1450-y
}

We read the article by Thelin et al. entitled "Utility of neuron-specific enolase in traumatic brain injury; relations to S100B levels, outcome, and extracranial injury severity" [1] with great interest. The authors conclude that while S100B and neuron-specific enolase (NSE) are both biomarkers for long-term outcome, S100B is a more accurate predictor.

These findings concur with the results of a previous study by our research group [2], which showed that serum S100B levels in severe traumatic brain injury (TBI) patients had a higher prognostic capacity to predict mortality than NSE. Similarly, most studies examining the role of diverse biomarkers in TBI pathology have identified S100B as the most promising, with the highest prognostic ability to predict short/long-term mortality [3, 4]. In recent years, researchers have searched for the ideal diagnostic biomarker that could guide TBI treatment in both primary and secondary phases. In TBI, the primary injury is caused by biomechanical damage and the secondary insult is the result of biochemical cascades triggered by damaged neurons, glial cells, and blood vessels. The early rise in biomarkers of secondary injuries could help physicians prevent, or at least reduce, the extent of this potential damage.
Currently, TBI management is guided by clinical histories and neuroimaging techniques. While these techniques may be advanced, they are more costly than serum analysis, involve exposure to ionizing radiations, and have certain limitations when assessing brain damage severity. The ideal biomarker would stratify patients based on their severity, identifying patients with poorer prognosis and greater need for treatment before the patient's condition worsens. In terms of the nature of the sample, the ideal biomarker could be detected in serum or urine (a waste fluid) in order to avoid cerebrospinal fluid extraction, as reflected in our findings on the role of urine S100B levels as an early predictor of mortality after severe TBI [5]. Yet, the variability of potential inclusion criteria in clinical analysis, and particularly in clinical trials, hinders the standardization of biomarker utility. Studies with such promising results, like that of Thelin et al. [1], encourage researchers to continue investigating TBI pathophysiology, to find the perfect biomarker for TBI assessment, or at least a set of biomarkers that together can reflect the diverse injury characteristics of TBI.

\section{Authors' response}

\section{Eric Peter Thelin, Emma Jeppsson, Bo-Michael Bellander and David W. Nelson}

Dear Drs. Rodríguez-Rodríguez and Egea-Guerrero

We appreciate that you took the time to read our recently published article in Critical Care [1].

We agree with the authors concerning the findings in their recent publication "S100B and neuron-specific enolase as mortality predictors in patients with severe

\footnotetext{
* Correspondence: jjegeaguerrero@gmail.com

${ }^{2}$ NeuroCritical Care Unit, Virgen del Rocío University Hospital, IBIS/CSIC/

University of Seville, Avda. Manuel Siurot s/n, 41013 Seville, Spain

Full list of author information is available at the end of the article
}

traumatic brain injury" [2], which unfortunately was not available to us when we prepared our manuscript. In addition to mortality, we looked at the different stages of the Glasgow Outcome Score and noted similar results where higher serum levels of both S100B and NSE correspond to a more unfavourable outcome.

However, we would also like to stress that further monitoring (after $72 \mathrm{~h}$ ) of unconscious TBI patients is of value, which we showed in a cohort of $n=250$ patients in whom secondary increases of S100B were correlated 
to newly developed radiological findings and subsequently a worse outcome [6]. Moreover, these secondary increases have been shown to aid in clinical decision making for these patients by other groups [7]. Drs. Rodríguez-Rodríguez and Egea-Guerrero mention urinary $\mathrm{S} 100 \mathrm{~B}$ as a potential marker of injury assessment, and while we appreciate additional compartments could be sampled, we would like to stress the need for further research in order to create valid thresholds and temporal patterns of release for these.

In unison with Drs. Rodríguez-Rodríguez and EgeaGuerrero, we stress that the field should continue to utilize biomarkers to better understand how to interpret and assess levels in bodily fluids and how they relate to injury severity and outcome. We suggest that future promising markers of brain tissue fate be compared with S100B, as it appears to have the most utility of the TBI markers studied to date, in order to determine how they can add additional information concerning outcome and assessment of injury.

\section{Abbreviations}

NSE: Neuron-specific enolase; TBI: Traumatic brain injury

\section{Authors' contributions}

ARR performed the literature review and wrote the initial draft of the manuscript. JJEG edited and rewrote portions of the manuscript. All authors read and approved the final manuscript.

\section{Authors' information}

ARR: Emergency Department, Virgen del Rocío University Hospital, IBIS/CSIC/ University of Seville, Spain. JJEG: NeuroCritical Care Unit, Virgen del Rocío University Hospital, IBIS/CSIC/University of Seville, Seville, Spain. Eric Peter Thelin, MD, PhD. Department of Clinical Neuroscience, Karolinska Institutet, Stockholm, Sweden. Emma Jeppsson, MD, Karolinska Institutet, Stockholm, Sweden. Bo-Michael Bellander, MD, PhD. Department of Clinical Neuroscience, Karolinska Institutet, Stockholm, Sweden. David W Nelson, MD, PhD. Department of Physiology and Pharmacology, Section of Anesthesiology and Intensive Care, Karolinska Institutet, Stockholm, Sweden.

\section{Competing interests}

The authors declare that they have no competing interests. The authors alone are responsible for the content and writing of the paper.

\section{Consent for publication}

The manuscript has been read and approved by all of the authors and each author believes that the manuscript represents honest work.

\section{Author details}

${ }^{1}$ Emergency Department, Virgen del Rocío University Hospital, IBIS/CSIC/ University of Seville, Seville, Spain. ${ }^{2}$ NeuroCritical Care Unit, Virgen del Rocío University Hospital, IBIS/CSIC/University of Seville, Avda. Manuel Siurot s/n, 41013 Seville, Spain.

Received: 28 September 2016 Accepted: 26 October 2016 Published online: 18 November 2016

\section{References}

1. Thelin EP, Jeppsson E, Frostell A, Svensson M, Mondello S, Bellander BM, et al. Utility of neuron-specific enolase in traumatic brain injury; relations to S100B levels, outcome, and extracranial injury severity. Crit Care. 2016;20:285

2. Rodriguez-Rodriguez A, Egea-Guerrero JJ, Gordillo-Escobar E, EnamoradoEnamorado J, Hernandez-Garcia C, Ruiz de Azua-Lopez Z, et al. S100B and neuron-specific enolase as mortality predictors in patients with severe traumatic brain injury. Neurol Res. 2016;38:130-7.

3. Gradisek P, Osredkar J, Korsic M, Kremzar B. Multiple indicators model of long-term mortality in traumatic brain injury. Brain Inj. 2012;26:1472-81.

4. Di Battista AP, Buonora JE, Rhind SG, Hutchison MG, Baker AJ, Rizoli SB, et al. Blood biomarkers in moderate-to-severe traumatic brain injury: potential utility of a multi-marker approach in characterizing outcome. Front Neurol. 2015;6:110.

5. Rodriguez-Rodriguez A, Egea-Guerrero JJ, Leon-Justel A, Gordillo-Escobar E, Revuelto-Rey J, Vilches-Arenas A, et al. Role of S100B in urine and serum as an early predictor of mortality after severe traumatic brain injury in adults. Clin Chim Acta. 2012;24:228-33.

6. Thelin EP, Nelson DW, Bellander BM. Secondary peaks of S100B in serum relate to subsequent radiological pathology in traumatic brain injury. Neurocrit Care. 2014:20(2):217-29.

7. Raabe A, Kopetsch O, Woszczyk A, Lang J, Gerlach R, Zimmermann M, Seifert V. S-100B protein as a serum marker of secondary neurological complications in neurocritical care patients. Neurol Res. 2004;26(4):440-5. 